Research Paper:

\title{
Comparing of the Effects of Acceptance and Commitment Therapy and Emotion Regulation Training on Diabetes Empowerment
}

\author{
Elham Eghrari $^{1}$ (D), Mohammad Hossein Bayazi $^{{ }^{*}}$ (D), Ali Reza Rajayi ${ }^{1}$
}

1. Department of Psychology, Torbat-e-Jam Branch, Islamic Azad University, Torbat-e-Jam, Iran.

\begin{tabular}{|c|c|}
\hline $\begin{array}{l}\text { Use your device to scan } \\
\text { and read the article online }\end{array}$ & ditetion: Elham, E., Bayazi, M. H., \& Rajayi, A. R., 2021. Comparing of the Effects of Acceptance and Commitment Ther- \\
\hline 口itit+a & $\begin{array}{l}\text { apy and Emotion Regulation Training on Diabetes Empowerment. Journal of Client-Centered Nursing Care, 7(1), pp. 55-64. } \\
\text { https://doi.org/10.32598/JCCNC.7.1.356.1 }\end{array}$ \\
\hline 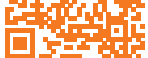 & dol'https://doi.org/10.32598/JCCNC.7.1.356.1 \\
\hline
\end{tabular}

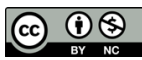

Article info

Received: 05 Mar 2020

Accepted: 11 Apr 2020

Published: 01 Feb 2021

Keywords:

Patient acceptance,

Emotional aspects,

Regulation,

Empowerment, Diabetes

\begin{abstract}
A B S T RA C T
Background: Diabetes mellitus is a widely diffused chronic condition that affects several aspects of patients' lives. The patient's life in this condition depends on diabetes management ability. Therefore, this study aimed to compare the effects of Acceptance and Commitment Therapy (ACT) and Emotion Regulation Training (ERT) on the diabetes empowerment of patients with type 2 diabetes.
\end{abstract}

Methods: This was a quasi-experimental study with a pre-test, post-test-follow-up and a control group design. Of the statistical population of patients referring to the Diabetes Prevention and Control Center of Mashhad Province, Iran, 45 subjects were recruited by convenience sampling method; they were randomly assigned into three groups of ACT, ERT, and control ( $n=15 /$ group). The study subjects were assessed using the Diabetes Empowerment Scale (F-DES-28) in the pre-test stage, after the intervention, and 3 months later. The obtained data were analyzed by repeated-measures Analysis of Variance (ANOVA) in SPSS V. 22.

Results: Both provided interventions presented a significant effect on the empowerment of the research subjects, compared to the controls $(\mathrm{P}<0.001)$. The effectiveness of ACT on the diabetes empowerment of patients was significantly higher than that of the ERT group $(\mathrm{P}=0.04)$.

Conclusion: It is recommended to use ACT and ERT to empower patients with type 2 diabetes.

\section{* Corresponding Author:}

Mohammad Hossein Bayazi, PhD.

Address: Department of Psychology, Torbat-e-Jam Branch, Islamic Azad University, Torbat-e-Jam, Iran.

Tel: +98 (915) 1679599

E-mail: bayazi123@gmail.com 


\section{Highlights}

- In this study, Acceptance and Commitment Therapy (ACT) and Emotional Regulation Training (ERT) were used to increase diabetes empowerment in patients with type 2 diabetes.

- The study findings indicated that ACT and ERT enhanced diabetes empowerment in the explored patients with type 2 diabetes.

- The effectiveness of ACT on diabetes empowerment of patients was significantly higher than that of ERT.

\section{Plain Language Summary}

Diabetic patients are daily involved with complex conditions. They need to manage their disease through medication and nutrition regimes. The stress associated with the disease and its complication diminishes their quality of life. In this regard, diabetes empowerment helps them to improve management ability. Acceptance and commitment therapy and emotion regulation training are therapeutic interventions applicable to increasing healthy emotions and improve the patient's ability to manage the disease. The study results indicated that acceptance and commitment therapy was more effective than emotion regulation training in diabetes empowerment.

\section{Introduction}

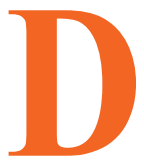

iabetes is associated with numerous serious biopsychological, familial, occupational, legal, and financial problems ( $\mathrm{Li}$ et al. 2018). Individuals with diabetes have reported poor mental health, lower health satisfaction, and life expectancy, as well as high levels of depression (Dehghani 2017). The prevalence of diabetes and pre-diabetes in Iranians aged $>35$ years is estimated to be approximately $18 \%$ and $34 \%$, respectively (Shamshirgaran et al. 2020). The annual worldwide diabetes-related mortality rate is about 53.6 per 100000 cases (Ling et al. 2020). A significant percentage of patients with diabetes experience low Quality of Life (QoL) (Waari, Mutai \& Gikunju 2018); most of them are not satisfied with medications and continuous regimen which put them under consistent stress. Therefore, treating these patients should consist of a comprehensive approach, including the patient's psychological condition. The recent psychological approaches attempted to improve chronic conditions by promoting psychological health and empowerment (Ghaedrahmati \& Jabalameli 2019; Yazdi, Saffarinia \& Zare 2020).

There are at least three reasons that justify psychological interventions for patients with diabetes, as follows: improving the disease and acceptance of the situation by the patient; behavioral changes for better self-care, and reducing the psychological barriers to control the disease (Rudaz et al. 2017). Psychological barriers can impair the illness perception and diminish treatment adherence. Accordingly, Acceptance and Commitment Therapy (ACT) can be a proper approach to improve the condition of these patients (Ghaedrahmati \& Jabalameli 2019). ACT is a third-wave cognitive behavioral therapy; unlike several therapies that emphasize reducing or controlling symptoms (e.g. negative thoughts \& emotions), it works when a direct change in the therapeutic condition is impossible (Pakenham 2017). Some researchers believe that mechanisms, such as acceptance, awareness, desensitization, and presence in the moment, non-judgmental observation in combination with conventional cognitive-behavioral techniques can increase self-care ability (Hor \& Manshaee 2017).This treatment was effective on the physical health of patients with diabetes (Ghaedrahmati \& Jabalameli 2019) Furthermore, emotions and feelings are directly associated with biopsychological health. Dysfunctional emotion regulation mechanisms reduce the ability of chronic disease management (Fisher et al. 2018). Emotion regulation is the ability to regulate emotions, modulate and regulate emotional experiences to achieve the desired emotional states and adaptive outcomes (Sobhi-Gharamaleki et al. 2015). Emotion Regulation Training (ERT) helps individuals to reduce negative emotions and learn to use positive strategies by increasing the ability to recognize emotions (Gross \& Thompson 2014). Patients with diabetes are always exposed to emotional problems due to fluctuations in glucose levels and problems in disease management; therefore, ER is critical in these patients (Ghiasvand \& Ghorbani 2015). ER ability decreases glucose levels in patients' blood serum (Yazdi et al. 2020) through self-care (Ghaedrahmati \& Jabalameli 2019). 
Therefore, the design and implementation of ER-based interventions could impact the improvement of diabetes empowerment. Various studies have highlighted the emotional and psychological dimensions in the management of diabetes (Yazdi et al. 2020). However, recent interventions, such as ERT and ACT have been rarely used in patients with diabetes. It is unclear whether the effects of these interventions on the level of diabetes empowerment of patients are the same; therefore, this study aimed to compare the effects of ACT and ERT on the diabetes empowerment of patients with type 2 diabetes.

\section{Materials and Methods}

This was a quasi-experimental study with a pre-test, post-test-follow-up and a control group design. The statistical population included all patients with diabetes who were referred to the Iranian Diabetes Prevention and Control Association of Mashhad Province, Iran from January 2020 to June 2020. In total, 45 subjects were selected based on the inclusion and exclusion criteria of the study. Subsequently, they were randomly assigned to 3 groups of control, ACT, and ERT (n=15/group).

The sample size was determined as 13 per group based on the following formula: the first type error of 0.05 ; the second type error of 0.20 , and considering $10 \%$ attrition. Accordingly, 16 patients were considered per group (Ghaedrahmati \& Jabalameli 2019).

$$
\begin{aligned}
& N=\underline{(\sigma 1+\sigma 2)^{2}} \underline{x} \times(z 1-\alpha / 2+z \beta)^{2} \\
& (x 1-x 2)^{2} \\
& (174) \times(0.84+1.96)^{2} / 105.06=12.9
\end{aligned}
$$

The inclusion criteria were as follows: at least high school education, the age range of 40-60 years, diagnosed with diabetes for $>2$ years, and no other physical and mental illnesses. Those who were absent from $>1$ session were excluded from this study.

The Diabetic Empowerment Scale (F-DES-28) was developed by Anderson et al. (2000). It consists of 28 items and 3 subscales, including psychological aspects of diabetes (9 questions), dissatisfaction and readiness for change (9 questions), and setting/achieving the goals of diabetes (10 questions). The questions of this tool are scored on a five-point Likert-type scale (strongly disagree $=1$, strongly agree $=5$ ). The minimum and maximum scores of this inventory range from 28 to 140 , with higher scores indicating greater empowerment in diabetes management.
Funnell and Anderson (2004) evaluated the reliability of the questionnaire. Accordingly, Cronbach's alpha coefficient was reported to be 0.81-0.96 for the total score and its components. The Persian version of F-DES was validated by Shojaeizadeh and associates (Shojaeezadeh et al. 2013). In the study of Nosratabadi et al. (2016), the internal consistency of this scale was computed as 0.74 0.96 for the total score and its subscales. Shojaeizadeh et al. (2013) confirmed the validity of the questionnaire through the factor analysis method.

A total of 69 patients with diabetes were evaluated. Furthermore, 48 subjects with type 2 diabetes who met the inclusion criteria of the study were recruited by convenience sampling technique. The selected cases were randomly assigned into ACT, ERT, and control groups ( $\mathrm{n}=16$ /group). Three participants were excluded due to unavailability in the follow-up stage (1 case), incomplete questionnaire (1 case), and absence from the sessions (1 case).

Pre-tests were performed by the researcher at the Diabetes Prevention Association Center. The F-DES-28 was completed after explaining the objectives of the study and signing informed consent forms by the research participants. Before the intervention, the research groups were examined and were homogeneous concerning age $(\mathrm{P}=0.27)$, gender $(\mathrm{P}=0.54)$, education $(\mathrm{P}=0.53)$, and diabetes empowerment score $(\mathrm{P}=0.47)$ (Table 1$)$.

The present study was performed during the Covid-19 outbreak; therefore, to prevent the infection risk of illness, the training sessions were conducted virtually via the Zoom platform in the form of video conferencing. Slides and group discussions were used to administer the research sessions. ACT Program formatted based on Hayes et al.'s (2004) study was presented in eight 90-minute weekly sessions (Hayes 2004). The sessions were adapted for patients with diabetes according to the study by Amsberg and colleagues (Amsberg et al. 2018) (Table 2).

This program was adapted for patients with diabetes according to the study by Chew and colleagues (Chew et al. 2017) (Table 2). Mean and standard deviation values were used to describe the collected data. Repeatedmeasures ANOVA was used to analyze the differences between time and groups. The level of significance was set at 0.05 .

\section{Results}

Table 3 presents the descriptive indexes of diabetes empowerment for the groups in pre-test, post-test, and follow-up phases. 
Table 1. Comparing the study groups' characteristics before the intervention

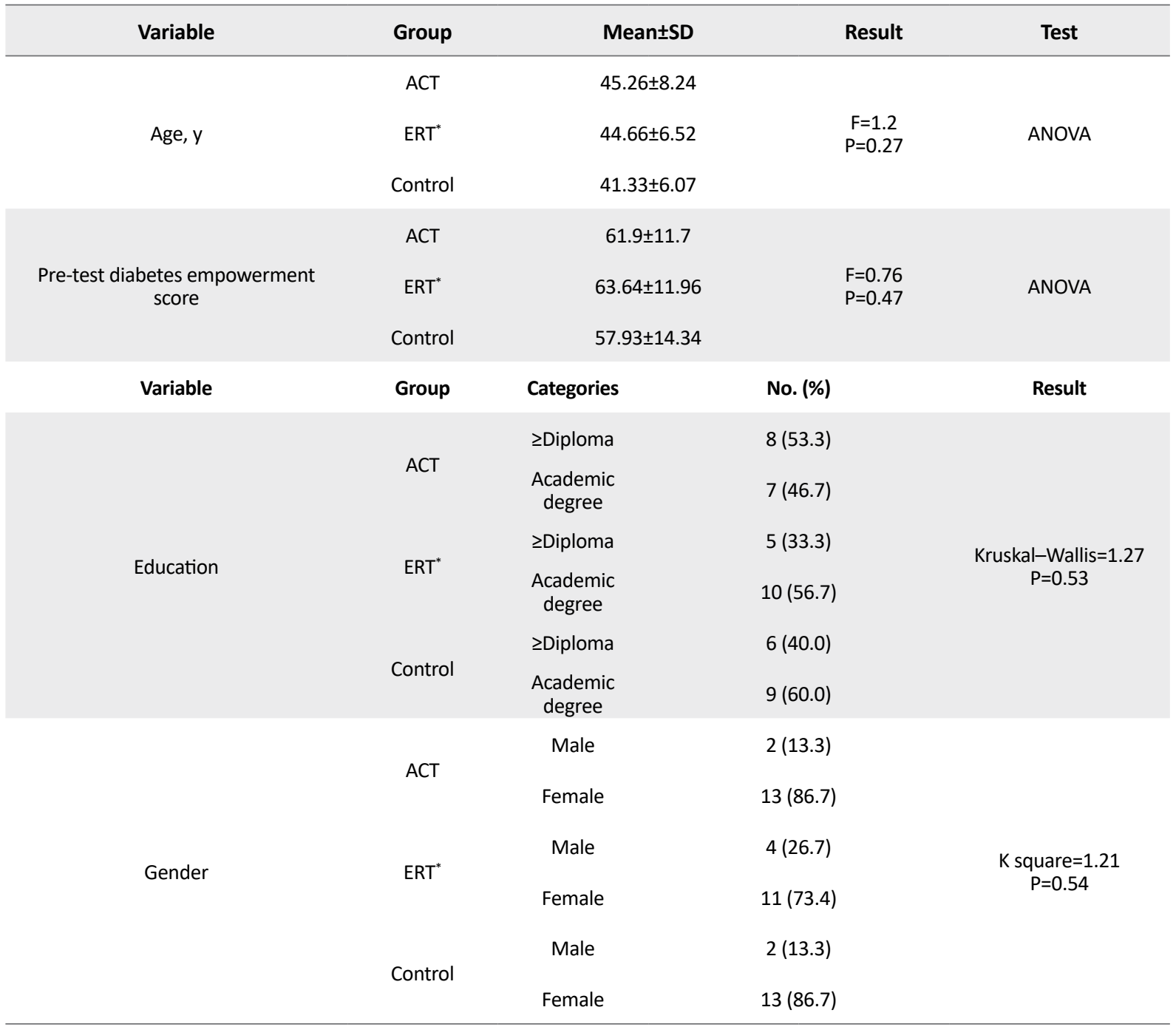

"Emotion Regulation Training.

Client- Centered Nursing Care

Table 3 presents an increase in the scores of diabetes empowerment from pre-test to post-test and follow-up in the intervention groups. The changes in the control group were minimal.

To investigate the pre-test, post-test-follow-up scores differences between the study participants, repeated-measures ANOVA was used. The results of Box's M test were not significant (Box's $\mathrm{M}=28.64, \mathrm{P}=0.10$ ); accordingly, the condition of the homogeneity of variance-covariance matrices was established. Moreover, the equal variance between the groups was checked by Levene's test, i.e. not significant. The results of Mauchly's sphericity test were also obtained (Mauchly=0.92, $\mathrm{x}^{2}=3.16, \mathrm{P}=0.20$ ).

Table 4 lists the results of repeated-measures ANOVA. Accordingly, time (pre-test, post-test, follow-up) significantly impacted diabetes empowerment in the study sub- jects. The significance of the time effect indicated a significant difference between the mean scores of pre-test, post-test, and follow-up in the ACT and wither, compared to the control group $(\mathrm{P}<0.001)$. The interaction of time and group was significant. The posthoc test results indicated that the mean score of the ACT group was higher than that of the ERT group $(\mathrm{P}<0.05)$. These achieved data indicated the effectiveness of ACT and ERT on diabetes empowerment in the study subjects. Bonferroni posthoc test was used to compare the pairs of the research groups (Table 5).

The relevant results revealed a significant difference in empowerment scores between the ACT and ERT groups. Moreover, ACT was significantly more effective than ERT in this respect $(\mathrm{P}<0.05)$. 
Table 2. The treatment session's contents

\begin{tabular}{|c|c|}
\hline Sessions & ACT (Amsberg et al. 2018) \\
\hline 1 & $\begin{array}{l}\text { Establishing a therapeutic relationship, an introduction to ACT, conducting pre-test, concluding a therapeutic contract } \\
\text { (What is possible to change and what needs to be accepted about diabetes disease?) }\end{array}$ \\
\hline 2 & $\begin{array}{l}\text { Stress and acceptance: How stress and emotions affect diabetes? Discovering and evaluating treatment methods and } \\
\text { evaluating their effectiveness, discussing the temporal and ineffectiveness of treatments using allegory, receiving feed- } \\
\text { back, and assigning homework. }\end{array}$ \\
\hline 3 & $\begin{array}{l}\text { How avoidance thoughts and behaviors can harm our physical health? Assisting clients with identifying ineffective disease } \\
\text { control strategies and realizing their futility, and accepting daily events without conflicts, using allegory, receiving feedback, } \\
\text { and assigning homework. }\end{array}$ \\
\hline 4 & $\begin{array}{l}\text { Values clarification: What is important in my life? How do I want to live? Explaining avoiding painful experiences and being } \\
\text { aware of the consequences of neglecting disease management, teaching steps to accept and change language concepts } \\
\text { using allegory, relaxation training, receiving feedback, and assigning homework }\end{array}$ \\
\hline 5 & $\begin{array}{l}\text { Explaining the concepts of role and context, observing the self as a platform and making contact with oneself using al- } \\
\text { legory, being aware of different sensory perceptions during daily diabetes management, and being aware of abilities and } \\
\text { capabilities. }\end{array}$ \\
\hline 6 & $\begin{array}{l}\text { Introducing a three-dimensional behavioral model to express the common relationship between behavior/emotions, psy- } \\
\text { chological functions, and observable behaviors, and discuss attempts to change diabetes management behavior based on } \\
\text { it, receive feedback, and assigning homework. }\end{array}$ \\
\hline 7 & $\begin{array}{l}\text { The acceptance of diabetes complications and programing for commitment to therapy, communication, and repetition } \\
\text { with diabetic patients to find approaches for daily management; explaining the concept of values, motivating the clients } \\
\text { for change and empowerment for a better life, practice concentration receive, feedback, and assigning homework. }\end{array}$ \\
\hline 8 & $\begin{array}{l}\text { Commitment to therapy and disease management training by practice, identifying behavioral plans as per values and creat- } \\
\text { ing a commitment to act on them, summarizing sessions, and conducting post-test. }\end{array}$ \\
\hline Sessions & ERT (Chew et al. 2017) \\
\hline 1 & $\begin{array}{l}\text { Introduction and familiarizing group members with each other, increasing motivation for treatment by examining the } \\
\text { advantages and disadvantages of changing the current situation and expressing treatment goals, emotional disturbance } \\
\text { and diabetes, emotion regulation, and biopsychological health. }\end{array}$ \\
\hline 2 & $\begin{array}{l}\text { Emotion, stress, diabetes: Providing education about the nature of emotions and the main components of emotion and } \\
\text { the concept of learned responses. }\end{array}$ \\
\hline 3 & $\begin{array}{l}\text { Teaching awareness to emotions and the evaluation of physical thoughts, feeling, and sensations (e.g. I cannot affect my } \\
\text { body through self-care, my body is different, diet is not effective for my body). }\end{array}$ \\
\hline 4 & $\begin{array}{l}\text { What are adaptive and maladaptive emotion regulation strategies? How adaptive strategies affect mental and physical } \\
\text { health? How maladaptive strategies lead to poor diabetes management? Awareness of the patterns of thinking about } \\
\text { the disease, flexibility, and changing cognitive evaluation. }\end{array}$ \\
\hline 5 & $\begin{array}{l}\text { Identifying and discussing methods for regulating negative and positive emotions, identifying the effectiveness of adap- } \\
\text { tive emotion regulation in diabetes therapeutic adherence. }\end{array}$ \\
\hline 6 & $\begin{array}{l}\text { How thinking can cause pain and suffering: The awareness of bodily emotions in emotional experiences to identify how } \\
\text { bodily emotions affect behaviors and thoughts. Awareness about the effectiveness of diabetes management strategies. }\end{array}$ \\
\hline 7 & $\begin{array}{l}\text { Attention bias control strategies, facing the internal and external triggers of emotion to increase tolerance for disease- } \\
\text { related emotions. }\end{array}$ \\
\hline 8 & $\begin{array}{l}\text { Reviewing the general concepts of therapy, examining the manners of continuing therapeutic achievements to prevent a } \\
\text { recurrence, and conducting post-test. }\end{array}$ \\
\hline
\end{tabular}

Client- Centered Nursing Care

\section{Discussion}

This study compared the effects of ACT and ERT on the diabetes empowerment of patients with type 2 diabetes. The obtained results revealed that ACT was effective in the enhancement of diabetes empowerment. Ghasemlou and Nezhadmohammad Nezhadm (2018) also explored the influence of group ACT on the QoL and anxiety among females with diabetes. They concluded that ACT could effectively improve the biopsychological health status of the study subjects. Besides, Nasiri et al. (2020) investigated the effects of group ACT on self-care and $\mathrm{HbA} \mathrm{C}$ in patients with type 2 diabetes. The collected results indicated that the level of $\mathrm{HbA} 1 \mathrm{C}$ significantly decreased and self-care behaviors improved in the study samples (Nasiri et al. 2020). 
Table 3. The mean and standard deviation scores of pre-test, post-test-follow-up stages in diabetes empowerment

\begin{tabular}{cccc}
\hline & & Mean \pm SD & \\
\cline { 2 - 4 } & ACT Group & ERT Group & Control Group \\
\hline Pre-test & $61.9 \pm 11.7$ & $63.64 \pm 11.96$ & $57.93 \pm 14.34$ \\
Post-test & $89.86 \pm 5.11$ & $80.66 \pm 10.70$ & $59.72 \pm 15.64$ \\
Follow-up & $97.00 \pm 12.51$ & $78.66 \pm 11.79$ & $59.33 \pm 17.00$ \\
\hline
\end{tabular}

Table 4. The results of repeated-measures ANOVA for the pre-test, post-test comparison and tracking of empowerment scores in the research groups

\begin{tabular}{ccccccc}
\hline Sources & SS & df & MM & F & P & Eta \\
\hline Time & 8157.64 & 2 & 4078.82 & 77.89 & 0.001 & 0.65 \\
\hline Time group & 4827.68 & 4 & 1206.92 & 23.04 & 0.001 & 0.52 \\
\hline Error & 4398.66 & 84 & 52.36 & & & 0.45 \\
Group & 13214.80 & 2 & 6607.40 & 17.43 & 0.001 & \\
Error & 15919.60 & 42 & 397.03 & & & \\
\hline
\end{tabular}

Increasing attention, emotional awareness, and practical desires are the positive aspect of ACT that leads to harmonizes adaptive behaviors and positive psychological states; even in improving the individual ability for following the individual and social activities. Improving psychological abilities and encouraging individuals to participate in the discussion and acceptance of experiences can be useful and effective in promoting their health (Rudaz et al. 2017). In the ACT, in addition to self-awareness and familiarity with regulating emotions, participants learn to commit to the therapy process for controlling the disease (LindholmOlinder et al. 2015). Reducing disease avoidance and its acceptance, along with the awareness of emotions, provide patients with the knowledge; through which, they can identify their feelings and emotional states and their relationship to physical states. This awareness results in the diminished negative effects of emotions through the management and acceptance of feelings; in ACT, patients learn to recognize values and adhere to make life meaningful in difficulties (Maghsoudi et al. 2019). In this study, the participants accepted that they have an incurable disease. Besides, in addition to recognizing and accepting their emotions, they had to make a commitment and effort to improve their situation; therefore, this method presented a greater effect on improving diabetes empowerment, compared to ERT.

Self-efficacy in diabetes management depends on the individual's emotional interpretation of events and facts (Benfer, Bardeen \& Clauss 2018). Improving the biopsychological condition of patients during the sessions gradually helped them to feel better. Subsequently, such feelings of improvement positively impacted various aspects of their self-efficacy for diabetes management (Rahimi, Nouri \& Raimi 2019). In this approach,

Table 5. The results of the Posthoc test

\begin{tabular}{|c|c|c|c|}
\hline & & Mean Difference & $\mathbf{P}$ \\
\hline \multirow{3}{*}{ ACT } & ERT & 8.66 & 0.04 \\
\hline & & & \\
\hline & Control & 23.93 & 0.001 \\
\hline \multirow[b]{2}{*}{ ERT } & ACT & -8.66 & 0.04 \\
\hline & Control & 15.26 & 0.001 \\
\hline
\end{tabular}

Client- Centered Nursing Care 
the patient is taught that any action to avoid or control these undesired mental experiences is ineffective or of the opposite effect. Besides, the experiences should be accepted completely, without any reaction to eliminate them. Accepting the disease condition builds an adaptive view toward the diabetes management process along with higher commitment (Gregg et al. 2007).

The study findings proved the effectiveness of group ERT on diabetes empowerment. In line with this study, Ghiasvand and Ghorbani (2015) assessed the effects of ERT on ER strategies and glycemic control in patients with type 2 diabetes; accordingly, they reported the effectiveness of ERT on glycemic level control (Ghiasvand \& Ghorbani 2015). According to Gross's (1998) agreement process model, emotions begin with the evaluation of the external and internal signs of emotion; subsequently, this evaluation leads to the coordination of a set of behavioral, experimental, physiological, and emotional responses. According to Gross's theory, individuals who reevaluate emotions experience, express higher positive emotions than those who restrain emotions (Gross \& Thompson 2014).

The obtained findings revealed a significant difference between the effects of ACT and ERT on diabetes empowerment in the explored patients with type 2 diabetes. In the group receiving ACT, the participants reported significantly higher diabetes empowerment. Consistent with this finding, Doroudian, Maddahi and Khal'atbary (2018) concluded that both approaches were effective in improving ER and self-efficacy in females with social anxiety disorder. ACT was more effective in improving mental health. However, Iri et al. (2015) argued that ACT was less effective in women's anxiety; this finding can be related to the differences between the variables and samples under study. Narimani et al. (2013) also reported that the effectiveness of ACT was higher than ERT among students. To justify this difference, we can address the mechanism of the effectiveness of these treatment programs. ACT uses a wide range of techniques in which in addition to facing emotions, the patient learns to accept his/her feelings and ultimately commits to treatment. Diabetes empowerment is a multidimensional variable involved with biopsychosocial components (Rahimi et al. 2019). Therefore, when multidimensional therapy is used, it is more effective than a therapy that only teaches emotional awareness and adaptation to emotion.

\section{Conclusion}

According to the present research findings, ACT was more effective than ERT in the empowerment of patients with type 2 diabetes. The present study targeted individuals with type 2 diabetes; thus, the results may differ in other populations or diseases. However, this study had some limitations. The most important of which was the coincidence of the COVID-19 outbreak; therefore, access to the samples and obtaining their cooperation was challenging. The researcher had limited sessions to online meetings. Additionally, the therapies were performed by the same researcher who might be more proficient in one approach, and this may have affected the achieved results. The cognitive and socio-psychological changes in societies are rapidly growing. Similarly, nursing and patient care has taken on new dimensions and nurses need to gather updated knowledge and skills for patient care. Therefore, nurses need to update their knowledge concerning psychological care through interdisciplinary training. This suggests the use of novel and different psychological approaches in modern nursing; accordingly, such measures can accelerate patient recovery and reduce hospitalization costs for patients and governments.

\section{Ethical Considerations}

\section{Compliance with ethical guidelines}

This study was approved by the Ethics Committee of Islamic Azad University, Torbat-e-Jam Branch (Code: IR.IAU.TJ.REC.1399.001). The necessary permissions were obtained and submitted to the Diabetes Prevention and Control Foundation of Mashhad Province. A written informed consent form was obtained from all study participants.

\section{Funding}

This article was extracted from the $\mathrm{PhD}$ dissertation of the first author at the Department of Psychology, Torbate-Jam Branch, Islamic Azad University, Torbat-e-Jam.

\section{Authors' contributions}

All authors equally contributed to preparing this article.

\section{Conflict of interest}

The authors declared no conflicts of interest. 


\section{Acknowledgments}

We would like to thank the managers and staff of the Iranian Diabetes Prevention and Control Association who cooperated with us to conduct the research.

\section{References}

Amsberg, S., et al., 2018. Acceptance and Commitment Therapy (ACT) for adult type 1 diabetes management: Study protocol for a randomised controlled trial. BMJ Open, 8(11), p. e022234. [DOI:10.1136/bmjopen-2018-022234] [PMID] [PMCID]

Anderson, R.M., et al., 2000. The Diabetes Empowerment Scale: A measure of psychosocial self-efficacy. Diabetes Care, 23(6), pp. 739-43. [DOI:10.2337/diacare.23.6.739]

Benfer, N., Bardeen, J. R., \& Clauss, K., 2018. Experimental manipulation of emotion regulation self-efficacy: Effects on emotion regulation ability, perceived effort in the service of regulation, and affective reactivity. Journal of Contextual Behavioral Science, 10, pp. 108-14. [DOI:10.1016/j.jcbs.2018.09.006]

Chew, B. H., et al., 2017. The effectiveness of a value-based emotion-cognition-focused educational programme to reduce diabetes-related distress in Malay adults with type 2 diabetes (VEMOFIT): Study protocol for a cluster randomised controlled trial. BMC Endocrine Disorders, 17(1), p. 22. [DOI:10.1186/s12902-017-0172-8] [PMID] [PMCID]

Dehghani, A., 2017. [Investigation of life style and quality of life in predicting depression in type II diabetic patients (Persian)] Journal of Diabetes Nursing, 5, pp. 320-31. http://jdn.zbmu. ac.ir/article-1-241-en.html

Doroudian, N., Maddahi, M., \& Khal'atbary, J., 2018. [A comparison of the impact of cognitive-behavioral therapy and acceptance and commitment therapy on the emotional regulation and self-efficacy of women with social anxiety (Persian)]. The Women and Families Cultural-Educational, 13(44), pp. 89-108. https://cwfs.ihu.ac.ir/article_203718.html?lang=en

Fisher, L., et al., 2018. Emotion regulation contributes to the development of diabetes distress among adults with type 1 diabetes. Patient Education and Counseling, 101(1), pp. 124-31. [DOI:10.1016/j.pec.2017.06.036] [PMID] [PMCID]

Funnell, M. M., \& Anderson, R. M., 2004. Empowerment and self-management of diabetes. Clinical Diabetes, 22, pp. 123-7. [DOI:10.2337/diaclin.22.3.123]

Ghaedrahmati, A., \& Jabalameli, S., 2019. [Effect of acceptance and commitment therapy on the quality of life and physical indices of patients with diabetes (Persian)]. Journal of Diabetes Nursing, 7(4), pp. 915-28. http://jdn.zbmu.ac.ir/article1-375-en.html

Ghasemlou, Z., \& Nezhadmohammad Nezhadm, A., 2018 Group training based on acceptance-commitment approach for improving quality of life and reducing anxiety among diabetic women. Journal of Research and Health, 8(6), pp. 513-21. [DOI:10.29252/jrh.8.6.513]
Ghiasvand, M., \& Ghorbani, M., 2015. [Effectiveness of emotion regulation training in improving emotion regulation strategies and control glycemic in type 2 diabetes patients (Persian)]. Iranian Journal of Endocrinology and Metabolism, 17(4), pp. 299-307. http://ijem.sbmu.ac.ir/article-1-1924-fa.html

Gregg, J. A., et al., 2007. Improving diabetes self-management through acceptance, mindfulness, and values: A randomized controlled trial. Journal of Consulting and Clinical Psychology, 75(2), pp. 336-43. [DOI:10.1037/0022-006X.75.2.336] [PMID]

Gross, J. J., \& Thompson R. A., 2014. Emotion regulation: Conceptual and empirical foundations. In: J. J. Gross (ed), Handbook of emotion regulation, The Guilford Press, New York.

Hayes, S. C., 2004. Acceptance and commitment therapy, relational frame theory, and the third wave of behavioral and cognitive therapies. Behavior Therapy, 35(4), pp. 639-65. [DOI:10.1016/S0005-7894(04)80013-3]

Hor, M., \& Manshaee, G. R., 2017. [Effectiveness of acceptance and commitment therapy on mental health of the patients with type 2 diabetes in the city of Isfahan (Persian)]. Iranian Journal of Diabetes and Lipid Disorders, 16(6), pp. 309-16. https://ijdld.tums.ac.ir/article-1-5566-en.html

Iri, H., et al., 2019. [Comparison of the effectiveness of Acceptance and Commitment Therapy and Dialectical Behavioral Therapy on health anxiety, psychosocial adjustment and cognitive emotion regulation of divorced women (Persian)] Medical Journal of Mashhad University of Medical Sciences, 61(1) pp. 79-88. [DOI:10.22038/MJMS.2019.13786]

Li, Y., et al., 2018. Time trends of dietary and lifestyle factors and their potential impact on diabetes burden in china. Diabetes Care, 41(5), p. 1116 [DOI:10.2337/dc18-er05] [PMID] [PMCID]

Lindholm-Olinder, A., et al., 2015. A randomised wait-list controlled clinical trial of the effects of acceptance and commitment therapy in patients with type 1 diabetes: A study protocol. BMC Nursing, 14, p. 61. [DOI:10.1186/s12912-015-0101-y] [PMID] [PMCID]

Ling, W., et al., 2020. Global trend of diabetes mortality attributed to vascular complications, 2000-2016. Cardiovascular Diabetology, 19(1). p. 182. [DOI:10.1186/s12933-020-01159-5] [PMID] [PMCID]

Maghsoudi, Z., et al., 2019. Efficacy of acceptance and commitment therapy for emotional distress in the elderly with type 2 diabetes: A randomized controlled trial. Diabetes, Metabolic Syndrome and Obesity: Targets and Therapy, 12, pp. 2137-43. [DOI:10.2147/DMSO.S221245] [PMID] [PMCID]

Narimani, M., et al., 2013. [A study comparing the effectiveness of acceptance/commitment by emotional regulation training on adjustment in students with dyscalculia (Persian)]. Journal of Learning Disabilities, 2(4), pp. 154-76. http://jld.uma.ac.ir/ article_139.html?lang=en

Nasiri, F., et al., 2020. [Effectiveness of group acceptance and commitment therapy (ACT) on self-care and $\mathrm{HbA1C}$ in type II diabetic patients (Persian)]. Feyz Journal of Kashan University of Medical Sciences, 24, pp. 209-18. http://feyz.kaums.ac.ir/ browse.php?a_id=3782\&sid=1\&slc_lang=en

Nosratabadi, M., et al., 2016. [Exploring the empowerment index and its related factors in type 2 diabetes patients in Sirjan city (Persian)]. Medical Journal of Mashhad University of Medical Sciences, 59(1), pp. 56-63. [DOI:10.22038/MJMS.2016.6990] 
Pakenham, K. I., 2017. Training in acceptance and commitment therapy fosters self-care in clinical psychology trainees. Clinical Psychologist, 21(3), pp. 186-94. [DOI:10.1111/cp.12062]

Rahimi, M., Nouri, R., \& Raimi, M., 2019. The effectiveness of the Acceptance and Commitment Therapy (ACT) approach on quality of life and hemoglobin A1c among patients with type 2 diabetes. International Journal of Medical Investigation, 8(2), pp. 61-9. http://intjmi.com/article-1-400-en.html

Rudaz, M., et al., 2017. Mindfulness and acceptance-based trainings for fostering self-care and reducing stress in mental health professionals: A systematic review. Journal of Contextual Behavioral Science, 6(4), pp. 380-90. [DOI:10.1016/j.jcbs.2017.10.001]

Shamshirgaran, S. M., et al., 2020. Prevalence of diabetes, prediabetes, awareness, treatment and control among people 35 years and up. Research Square, 1, pp. 26-38. [DOI:10.21203/ rs.3.rs-22638/v1]

Shojaeezadeh, D., et al., 2013. [Effect of education program based on empowerment model in promoting self-care among type 2 diabetic patients in Isfahan (Persian)]. Razi Journal of Medical Sciences, 20(107), pp. 18-31. http://rjms.iums.ac.ir/browse. php?a_id $=2547 \&$ sid $=1 \&$ slc_lang $=$ en

Sobhi-Gharamaleki, N., et al., 2015. [Effectiveness of emotion regulation training on reduction of anxiety, stress and depression symptoms among university students (Persian)]. Iranian Journal of Health Education \& Promotion, 3(1), pp. 5-13. http:// journal.ihepsa.ir/article-1-234-en.html

Waari, G., Mutai, J., \& Gikunju, J., 2018. Medication adherence and factors associated with poor adherence among type 2 diabetes mellitus patients on follow-up at Kenyatta National Hospital, Kenya. Pan African Medical Journal, 29, p. 82. [DOI:10.11604/pamj.2018.29.82.12639] [PMID] [PMCID]

Yazdi, M., Saffarinia, M., \& Zare, H. 2020. [Comparison the efficacy of life-based treatment and emotional regulation therapy on $\mathrm{HbA1C}$ biological markers and blood glucose in patients with type 2 diabetes (Persian)]. Quarterly Journal of Health, 9(1), pp. 61-78. http://hpj.journals.pnu.ac.ir/article_6688. html?lang=en 
This Page Intentionally Left Blank 